\title{
Exercise Induces Cortical Plasticity after Neonatal Spinal Cord Injury in the Rat
}

\author{
Tina Kao, ${ }^{1}$ Jed S. Shumsky, ${ }^{1}$ Marion Murray, ${ }^{1}$ and Karen A. Moxon ${ }^{1,2}$ \\ ${ }^{1}$ Department of Neurobiology and Anatomy, Drexel University College of Medicine, Philadelphia, Pennsylvania 19129, and ${ }^{2}$ School of Biomedical \\ Engineering, Science and Health Systems, Drexel University, Philadelphia, Pennsylvania 19104
}

\begin{abstract}
Exercise-induced cortical plasticity is associated with improved functional outcome after brain or nerve injury. Exercise also improves functional outcomes after spinal cord injury, but its effects on cortical plasticity are not known. The goal of this investigation was to study the effect of moderate exercise (treadmill locomotion, $3 \mathrm{~min} / \mathrm{d}, 5 \mathrm{~d} / \mathrm{week}$ ) on the somatotopic organization of forelimb and hindlimb somatosensory cortex (SI) after neonatal thoracic transection. We used adult rats spinalized as neonates because some of these animals develop weight-supported stepping, and, therefore, the relationship between cortical plasticity and stepping could also be examined. Acute, single-neuron mapping was used to determine the percentage of cortical cells responding to cutaneous forelimb stimulation in normal, spinalized, and exercised spinalized rats. Multiple single-neuron recording from arrays of chronically implanted microwires examined the magnitude of response of these cells in normal and exercised spinalized rats. Our results show that exercise not only increased the percentage of responding cells in the hindlimb SI but also increased the magnitude of the response of these cells. This increase in response magnitude was correlated with behavioral outcome measures. In the forelimb SI, neonatal transection reduced the percentage of responding cells to forelimb stimulation, but exercise reversed this loss. This restoration in the percentage of responding cells after exercise was accompanied by an increase in their response magnitude. Therefore, the increase in responsiveness of hindlimb SI to forelimb stimulation after neonatal transection and exercise may be due, in part, to the effect of exercise on the forelimb SI.
\end{abstract}

\section{Introduction}

Exercise induces changes in somatotopic maps of the somatosensory or motor cortices of animals with brain or peripheral injuries, and these changes are related to functional recovery (Friel et al., 2000; Florence et al., 2001; Ramanathan et al., 2006). In the case of spinal cord injury (SCI), exercise also improves recovery, but the role of exercise-induced cortical plasticity after SCI is not well understood (Weidner et al., 2001; Cai et al., 2006).

SCI alone can alter somatotopic maps in two ways. The first is sometimes referred to as "silencing." This refers to the case when the region of the somatosensory cortex most affected by the injury does not respond to any cutaneous stimulation (Jain et al., $1995,2003)$. The second possibility is that the affected region develops a novel somatotopic organization, such that the cells in this region respond to stimulation of peripheral areas innervated from spinal segments rostral to the injury (McKinley and Swyter, 1989; Chau and McKinley, 1991). The influences of postinjury behavioral activity of the animals in these studies, however, were rarely addressed. Jain et al. (2003) examined somatosensory

Received June 2, 2008; revised April 8, 2009; accepted April 14, 2009.

This work was supported by National Institutes of Health Grant P01 NS24707. We thank Dr. Simon Giszter for access to some of the rats used in this study and Dr. Tim Himes and Theresa Connors for assistance in the transections. We also thank Nicole Amato for her assistance in the exercise protocols and Dr. Jon Kaas for his input on the histology.

Correspondence should be addressed to Dr. Karen A. Moxon, School of Biomedical Engineering, Science and Health Systems, Drexel University, 3141 Chestnut Street, Philadelphia, PA 19104. E-mail: km57@drexel.edu.

T. Kao's present address: Department of Neurobiology, The University of Chicago, 947 East 58th Street, Chicago, IL 60637 .

D01:10.1523/JNEUROSCI.2474-08.2009

Copyright $\odot 2009$ Society for Neuroscience $\quad 0270-6474 / 09 / 297549-09 \$ 15.00 / 0$ maps of spinalized rats exposed to an enriched environment and compared these to maps of spinalized rats exposed to standard laboratory housing. They found no differences in the response of cells in the affected somatosensory cortex between the two groups. In contrast, neurons in the affected somatosensory cortex of spinalized kittens that received passive exercise responded to sensory stimulation of peripheral areas innervated rostral to the injury (Chau and McKinley, 1991). Therefore, exercise may modify the organization of the somatosensory cortex after spinal cord injury.

Our goal was to examine the effects of exercise on organization of the hindlimb and forelimb somatotopic representations in the cortex in adult rats with neonatal midthoracic transections. Using acute, single-neuron mapping techniques (McKinley and Swyter, 1989; Jain et al., 1995; Leiser and Moxon, 2006), we first compared the percentage of cells in the supragranular, granular, and infragranular layers of the hindlimb somatosensory cortex that responded to sensory stimulation of forelimbs among three groups of rats: normal adult, nonexercised spinalized, and exercised spinalized rats. We also made quantitative measures of the magnitude of the response of single neurons in the hindlimb infragranular cortex of normal adults and exercised spinalized rats to the sensory stimulation (Foffani et al., 2004; Tutunculer et al., 2006; Leiser and Moxon, 2007; Moxon et al., 2008). Second, we evaluated the effect of exercise on cells in the forelimb somatotopic representation of the somatosensory cortex using both the acute and quantitative measures. Our results demonstrate that exercise not only increased the percentage of cells in the hindlimb somatosensory cortex of spinalized rats that responded 
to stimulations of the forelimbs, but exercise also increased the response magnitude of these cells. This increase in response magnitude was correlated with the frequency of weight-supported stepping on the treadmill. Exercise also increased the response magnitude of cells in the forelimb cortex of spinalized rats, which may have contributed to the enhanced responsiveness of cells in the hindlimb cortex.

\section{Materials and Methods}

\section{Overview of experimental methods}

To assess the percentage of responding cells in somatosensory cortex, an acute, single-neuron-mapping study was performed on normal adult rats, adult rats spinalized as neonates (spinalized), and adult rats spinalized as neonates given daily treadmill exercise (exercised spinalized). Single, microelectrodes were inserted into the forelimb or hindlimb primary somatosensory cortex, and single neurons from the supragranular, granular, and infragranular layers were identified as either responsive or not responsive to cutaneous stimulation of the forelimbs. Differences in the average percentage of cells that responded to cutaneous stimulation between the three groups (normal, spinalized, and exercised spinalized) were assessed using a one-way ANOVA and Tukey post hoc analysis. For groups that were determined to be significantly different from each other, we then assessed differences in the percentage of responding cells for each layer independently. The two-proportions test was used to assess difference between the ratio of responding cells to cells recorded for each cortical layer between the different animal groups.

To assess the effect of exercise after neonatal spinal transection on the output layer of the somatosensory cortex, chronic multiple, singleneuron recordings were made from the infragranular layer in normal adult and exercised spinalized rats, whereas cutaneous stimulation was applied to the forelimbs. For each animal, 16-channel microwire arrays were implanted bilaterally into the infragranular somatosensory cortex of the hindlimb or forelimb representation. Action potentials from single neurons in response to triggered sensory stimulation of shoulder and forelimbs were recorded, and peristimulus time histograms (PSTHs) were generated. The average number of spikes per stimulus and the latency of the response were compared between the two groups using a multivariate ANOVA (MANOVA). Differences between groups on individual variables were assessed using Tukey's post hoc test. In addition, the magnitude of the response of infragranular cells recorded from the hindlimb somatosensory cortex was correlated to the behavioral outcome of the animals. All surgical procedures were performed under the guidelines of the National Institutes of Health and approved by the Institutional Animal Care and Use Committee of Drexel University.

\section{Transection procedures}

The transection procedure for the Sprague Dawley pups was identical to our previous method (Shumsky et al., 2005; Kao et al., 2006). Briefly, Sprague Dawley (Charles River) pups were anesthetized by hypothermia, the spinal cord was exposed by laminectomy at the T8/T9 level, and transected with iridectomy scissors followed by aspiration on postnatal day 2. A collagen matrix, vitrogen, was injected into the site of the transection to fill the cavity. The muscle and skin were sutured in layers with 5-0 silk. Pups were then warmed, and when they became active, were returned to the mothers and littermates. The pups were weaned at 4 weeks and housed under a $12 \mathrm{~h}$ light/dark cycle with ad libitum access to food and water.

\section{Animals}

Spinalized rats were randomly divided into two groups, those that received exercise (exercised spinalized) and those that did not (spinalized). To ensure that the behavior of the two groups of rats was similar, rats were tested to assess their ability to support their weight during treadmill locomotion 4 weeks after spinalization, before exercise began for the exercise group. Some spinalized rats can support their weight during a step cycle during treadmill locomotion (Miya et al., 1997; Giszter et al., 1998; Kao et al., 2006). A step cycle was defined as a sequential flexion and extension of the hindlimb on the treadmill. Not all step cycles on a treadmill involved weight-supported stepping. Weight-supported step cycles, in which the hindlimbs support the hindquarters so that they are elevated above the surface of the treadmill, were distinguished from nonweight-supported step cycles, in which the hindlimbs flex and extend, but the knee remains in contact with the treadmill, and the hindquarters are not elevated above the surface of the treadmill. Treadmill sessions were videotaped in the lateral view, and locomotion was evaluated. The percentage of weight-supported steps (\%WSS) was assessed over 3 min of treadmill locomotion. The average weight of the exercisedspinalized rats (weight $=230 \pm 20 \mathrm{~g}$ ) was not different from the average weight of the spinalized rats (weight $=238 \pm 23 \mathrm{~g}$ ) when measured at the time of cortical recordings ( $p=0.70)$.

\section{Exercise protocol}

Spinalized rats that did not receive exercise were left sedentary in their cages but handled and checked $5 \mathrm{~d}$ /week for skin lesions and other health concerns. Starting 4 weeks after transection, rats in the exercise group were placed on a motorized treadmill apparatus for $3 \mathrm{~min} / \mathrm{d}$ at a speed of $6.5 \mathrm{~m} / \mathrm{min}, 5 \mathrm{~d}$ per week. No other stimulation was applied to the rats during treadmill locomotion. The exercise protocol continued for 6-8 months to be consistent with previous studies (Jain et al., 2003). Since the uninjured rats were freely moving in their cages, the regular locomotion undergone by the spinalized rats during this exercise protocol was an attempt to increase the activity level of the spinalized rats to a level comparable with that of normal rats.

\section{Acute single-unit-mapping procedure}

Acute single-neuron mappings were performed in three groups: normal controls, neonatally spinalized rats that did not receive exercise, and neonatally spinalized rats that received treadmill exercise. After spinalization ( $6-8$ months), rats were anesthetized by intraperitoneal injection of sodium pentobarbital $(45 \mathrm{mg} / \mathrm{kg})$ and placed in a stereotaxic frame. For all animals, the anesthesia level was maintained at Stage III-4 (Friedberg et al., 1999). This had the effect of reducing the responsiveness of cells, and we, therefore, continuously stimulated the cutaneous surface throughout the mapping procedure to identify single neurons (see below). Craniotomies were performed over either the right or left cortex to expose the hindlimb or forelimb representations in the primary somatosensory cortex. The stereotaxic coordinates for hindlimb craniotomy were from 0 to $3 \mathrm{~mm}$ posterior to bregma and from 2 to $3 \mathrm{~mm}$ lateral. The coordinates for forelimb craniotomy were from $1.5 \mathrm{~mm}$ anterior to $3 \mathrm{~mm}$ posterior and 3 to $5 \mathrm{~mm}$ lateral. Electrode penetrations were defined using the stereotaxic coordinates for the forelimb and hindlimb somatosensory cortex (Paxinos and Watson, 2007).

A high-impedance (10 MOhm) tungsten microelectrode (FHC) was mounted on a stereotaxic electrode manipulator. A ground wire was inserted into the brain adjacent to the craniotomies. The microelectrode was then moved to the anterior-posterior and medial-lateral coordinates that defined a predetermined location above either the forelimb or hindlimb somatosensory cortex (for electrode penetration locations, see Fig. 1) and lowered, perpendicular to the surface of the brain, to penetrate the dura and pia. The microelectrode was then slowly inserted into the brain.

The signals from the microelectrode were continuously monitored on the oscilloscope and audio speakers as the electrode was lowered. The cutaneous surface of the animal was continuously stimulated, whereas the electrode was lowered. When a neuron was encountered, the dorsal/ ventral coordinates of the cell were noted. Two experimenters then determined whether the identified cell responded to sensory stimulation. The first experimenter, with knowledge of the electrode placement, used wooden probes to touch the hair/skin on the forelimb and shoulder. The second experimenter, blind to the position of the electrode and treatment group of the animal, determined if the cell responded to the stimulus, predominately by listening for a change in firing rate. If the cell did not respond to the stimulation with an increase in firing rate, the cell was noted as negative. If the cell did respond to the stimulation with an increase in firing rate, the cell was noted as positive. If the cell was noted as positive, then the receptive field of the cell was identified by tapping locations on the body rostral to the level of the injury. Stimulation of any body surface that elicited an increase in firing was considered part of the cell's receptive field. To ensure that tapping forces between animals and 
A.

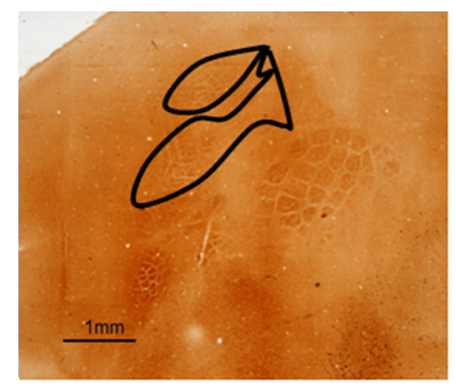

C.

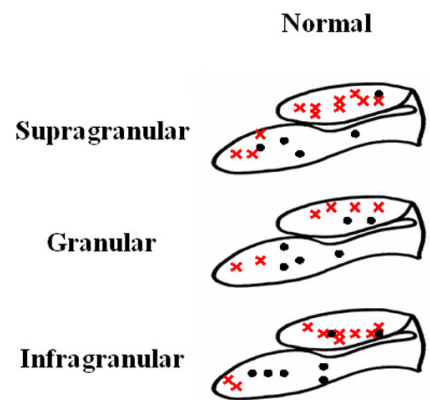

B.

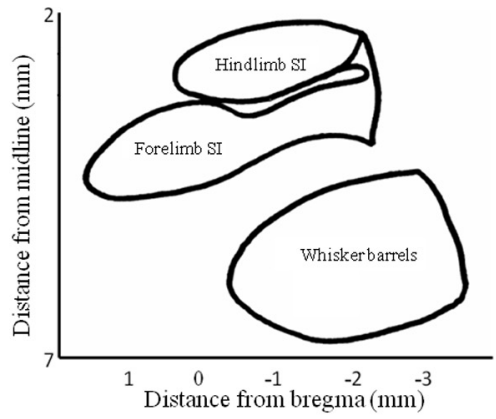

Spinalized
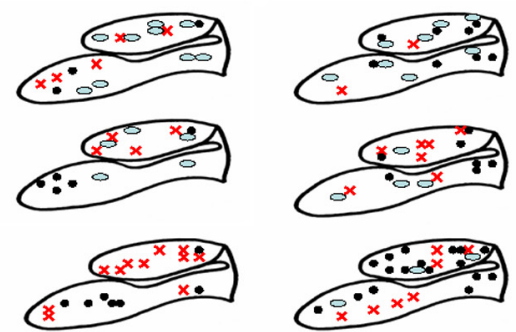

D.

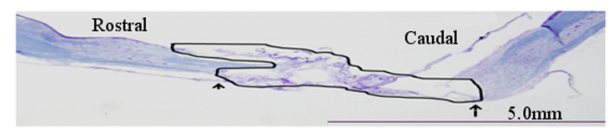

Figure 1. Penetration sites for the acute single-neuron mapping of forelimb and hindlimb somatosensory cortex in normal, spinalized, and exercised spinalized rats. $\boldsymbol{A}$, The flattened somatosensory cortex, when stained with cytochrome oxidase in a normal adult rat, reveals the locations that we targeted in this study. Solid lines outline the hindlimb and forelimb somatosensory cortices. $\boldsymbol{B}$, Schematic of the flattened somatosensory cortex based on $1 \mathrm{~mm}$ grids showing the stereotaxic coordinates of the forelimb and hindlimb somatosensory cortex. C, Penetration sites into the forelimb and hindlimb somatosensory cortex for each group of rats. Responses were recorded from electrodes placed in the supragranular, granular, and infragranular layers of the hindlimb and forelimb somatosensory cortices. Black circles, Electrode penetration sites that elicited cells which responded to sensory stimulation. Red $x$, Electrode penetration sites that elicited cells which did not respond to sensory stimulation. Ovals, Electrode penetration sites that did not elicit any cells. $\boldsymbol{D}$, Niss//myelin staining of the spinal cord of a representative adult rat transected as a neonate at the T8/T9 level. No cell bodies or axons were observed in the transection site (outlined in black). The extent of this representative injury was comparable with the other injuries. The average rostral to caudal extent of the injury was $6.4 \pm 2.2 \mathrm{~mm}$, and no differences were observed in the extent of the injury between the spinalized and exercised spinalized groups. Scale bar, $5.0 \mathrm{~mm}$.

across sites were uniform, the responses elicited by the wooden probe were periodically compared with responses elicited by von Frey filaments to calibrate the stimulus applied by the wooden probe. The stimulation consisted of pressing a filament gently against the skin, perpendicular to its surface until the filament bended $90^{\circ}$. This procedure was done five times for each filament and skin site, to ensure reproducibility of the results. The filament necessary to elicit a response similar to the wooden probe was noted and compared across animals and locations. The filaments required to produce an equivalent response ranged from 4.31 (bending force of $2 \mathrm{~g}$ ) to 4.93 (bending force of $8 \mathrm{~g}$ ) across animals and animal groups. There were no identifiable differences in the distribution of filaments used between the groups.

After a cell was characterized, the microelectrode was moved at least 50 $\mu \mathrm{M}$ deeper (with respect to the cortical surface) before another cell could be identified in the same penetration to ensure a new cell was encountered. For every cell identified, we determined if the cell responded to the stimulation of the forelimbs. In addition, the stereotaxic coordinates of the microelectrode position were identified, allowing us to evaluate neuronal responsiveness for each layer of the cortex. To minimize tissue damage and its possible effects on cell responsiveness during later penetrations, no more than seven penetrations were performed per animal. Approximately 20 cells were encountered during each penetration.

\section{Data analysis}

First, to determine if there were differences among the groups, the percentage of responding cells were noted for each penetration, and a one-way ANOVA was performed with Tukey post hoc test to identify significant differences between groups. For those groups for which a difference was identified, differences were then assessed on a layer-by-layer basis. Cells were assigned to different cortical layers based on penetration depth into the following: supragranular, 50-700 $\mu \mathrm{M}$ (layer II/III); granular, 750-1000 $\mu \mathrm{M}$ (layer IV); and infragranular, 1050-2000 $\mu \mathrm{M}$ (layer V) categories. The percentage of cells that responded to cutaneous stimulation rostral to the injury (positive) was defined as the number of cells responding to sensory stimulation over the total number of cells identified (positive and negative). This percentage was calculated for each layer of the cortex. The two-proportions test was used to assess difference between the ratio of responding cells to cells recorded for each cortical layer. The probability level for each group determined by the Fisher's exact test was used to test significance with a value of $p<0.05$ considered significant.

\section{Chronic multiple \\ single-neuron-recording procedure}

To determine if exercise also altered the response magnitude of these cells to cutaneous stimulation, triggered stimuli were applied across the forelimb cutaneous surface in a second set of animals that were chronically implanted with arrays of microelectrodes into the output or infragranular layer. The responsiveness of neurons in the hindlimb and forelimb representations of the somatosensory cortex of spinalized that received exercise was quantified using PSTHs and compared with those of normal rats. Rats were implanted with two microelectrode arrays, one into the left somatosensory cortex and the other into the right somatosensory cortex. After spinalization and exercise (6-8 months), rats were anesthetized by intraperitoneal injection of sodium pentobarbital $(45 \mathrm{mg} / \mathrm{kg})$ and placed in a stereotaxic frame. Craniotomies were performed over both the right and left cortices to expose the hindlimb or forelimb representations in the primary somatosensory cortex (Fig. $1 B$ ). The dura and pia were removed just before electrode implantation. Both electrode arrays consisted of two rows of eight $50 \mu \mathrm{M}$ Teflon-coated stainless steel microwires (NB labs) and were implanted one at a time. The electrode array was mounted on a micromanipulator, and a ground wire was inserted into the brain. The electrode array was aligned to bregma and oriented so that the rows ran rostrocaudally. The array was then moved to predetermined anterior/ posterior and medial/lateral coordinates within the exposed hindlimb and forelimb craniotomies. Once the array was placed at the predetermined coordinates, it was lowered perpendicular to the surface of the brain. As the electrode array was slowly lowered, the signals were monitored, one channel at a time, on the oscilloscope and audio speakers. When characteristic large amplitudes of infragranular neurons were recorded on the majority of electrodes, the array was cemented in place with dental cement. Animals were then allowed $10 \mathrm{~d}$ for any initial reaction to the implant to subside before the mapping sessions were performed.

After the $10 \mathrm{~d}$ recovery period, the mapping procedure was performed. Rats were lightly anesthetized with a low dose of Nembutal $(30 \mathrm{mg} / \mathrm{kg})$ 
and maintained in a lightly anesthetized state, Stage III-2 (Friedberg et al., 1999) to ensure minimal effect on neuronal responsiveness while immobilizing the rat. Signals from the electrodes were amplified and filtered with a multineuron acquisition system (Plexon). The resulting signals were displayed on an oscilloscope and amplified through loudspeakers to aid in online neuronal spike sorting on all channels corresponding to the electrodes.

Quantitative measures of neuronal responses were generated by recording the spike times of each neuron, whereas different locations across the body were repeatedly stimulated with an electronically controlled probe similar to our previous studies (Foffani et al., 2004; Tutunculer et al., 2006). Five locations on each side of the body were repeatedly contacted. The left and right forepaw digit 2 were contacted on the ventral surface in the center of the pad. The left and right forepaw were contacted on the dorsal surface in the center. The left and right shoulder were contact on the dorsal surface at the top of the humerus. The left and right forepaw palm pads 5 were contacted in the center on the ventral surface. Finally, the left and right lower arm were contacted on the dorsal surface midway along the radius/ulna.

Each of the above locations were consecutively stimulated 100 times at $0.5 \mathrm{~Hz}$ with a finetipped metal probe $1 \mathrm{~mm}$ in diameter. To be consistent with previous studies in healthy animals (Foffani et al., 2004; Tutunculer et al., 2006; Moxon et al., 2008) and ensure that only tactile receptors at the sight of contact were activated, the metal probe was controlled with an electromechanical stimulator (Gemini GV6), which delivered squared-pulse stimuli (duration, $100 \mathrm{~ms}$; frequency, $0.5 \mathrm{~Hz}$ ), similar to previous studies (Chapin, 1986; Foffani et al., 2004). The tip of the metal probe moved $0.5 \mathrm{~mm}$ in response to the square-pulse stimuli. To control the magnitude of the stimuli at each location, the metal probe was first positioned on the skin, ensuring contact but no visual indentation under $10 \times$ magnification. The metal probe was then moved $0.5 \mathrm{~mm}$ away from the skin, and the stimulation was started. This procedure allowed consistent stimulus across locations and across animals.

All locations were stimulated during the same recording session to ensure that the same neurons were recorded during stimulation of all 10 locations. The waveforms and action potential times of all the discriminated neurons were recorded and data stored in NeuroExplorer (Nex Technologies; version 2.66) for off-line analysis. For every stimulus location, PSTHs of all neurons were calculated using Nex functions and exported to Matlab (version 6.5; The Mathworks) for further analysis.

Data analysis. To identify significant responses in the PSTHs, three tests were performed for each discriminated neuron and peripheral location stimulated: (1) A threshold was set as the average background activity of the neuron (evaluated from $100 \mathrm{~ms}$ to $5 \mathrm{~ms}$ before the stimulus) plus three SDs, and the first and the last significant bin ( $1 \mathrm{~ms}$ bin size) that exceeded the threshold in a window between $5 \mathrm{~ms}$ and $90 \mathrm{~ms}$ after the stimulus was identified; (2) at least three bins were suprathreshold; and (3) the response between the first and last significant bin has to be significantly greater than the average background activity (nonpaired $t$ test, $p<0.001)$. For every significant response, three parameters were extracted from the PSTH: (1) response magnitude defined as the average number of spikes per stimulus between the first and the last significant bin minus the background firing rate (i.e., probability of spike per stimulus); (2) the first bin latency or the time interval between the stimulus onset and the first significant bin of the response; and (3) the last bin latency or the time interval between the stimulus onset and the last sig- nificant bin of the response. These three parameters (response magnitude, first bin latency, and last bin latency) were compared between groups (normal and spinalized exercised) using a MANOVA to assess differences between groups.

Data analysis for correlating percentage WSS and neuronal activity. Spearman R (Statistica 5.5; Statsoft), a nonparametric correlation test, was used to correlate the response magnitude of cells obtained from the chronic multiple single-unit recordings in the hindlimb and forelimb representations of the somatosensory of exercised, spinalized rats with individual measures of \%WSS. A $p$ value of $<0.05$ was considered significant.

Perfusion and histological processing of the brain and spinal cord At the end of the mapping sessions, the rats were perfused transcardially with buffered saline, followed by buffered $2 \%$ paraformaldehyde, and then by buffered $2 \%$ paraformaldehyde containing $10 \%$ sucrose. The cortex was removed and flattened between two glass slides. The tissue was cryoprotected in $30 \%$ sucrose and sectioned $(70 \mu \mathrm{M}$ parallel to the pial surface) frozen on a sliding microtome. Series of these sections from the cortex were stained for cytochrome oxidase activity (Wong-Riley, 1979). Spinal cords were removed and placed in phosphate buffer containing $30 \%$ sucrose for $72 \mathrm{~h}$. Specimens were frozen in Tissue-Tek O.C.T. compound (Sakura Finetek) and sectioned on a freezing microtome at 20 $\mu \mathrm{m}$. The transection segments of the spinal cords were sectioned parasagitally, and alternate sections were Nissl-myelin stained. The resulting sections were examined under a microscope to confirm completeness of the transection (Fig. 1D). The average extent of the lesion for all animals was $6.4 \pm 2.2 \mathrm{~mm}$, and there were no differences in the rostrocaudal extent of the lesions between the spinalized and the exercised spinalized groups.

To further ensure complete transections, animals with the best \%WSS 
A.

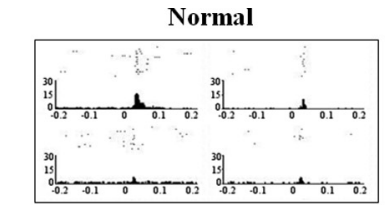

FPL2

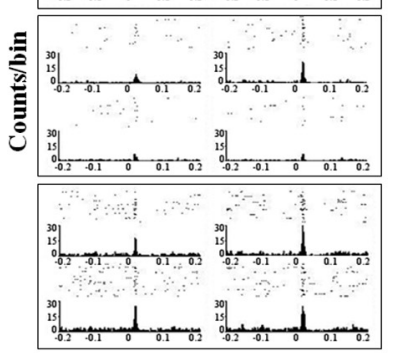

Time (sec)

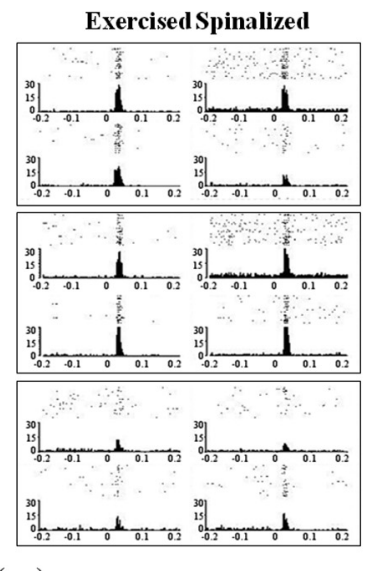

C. First Bin
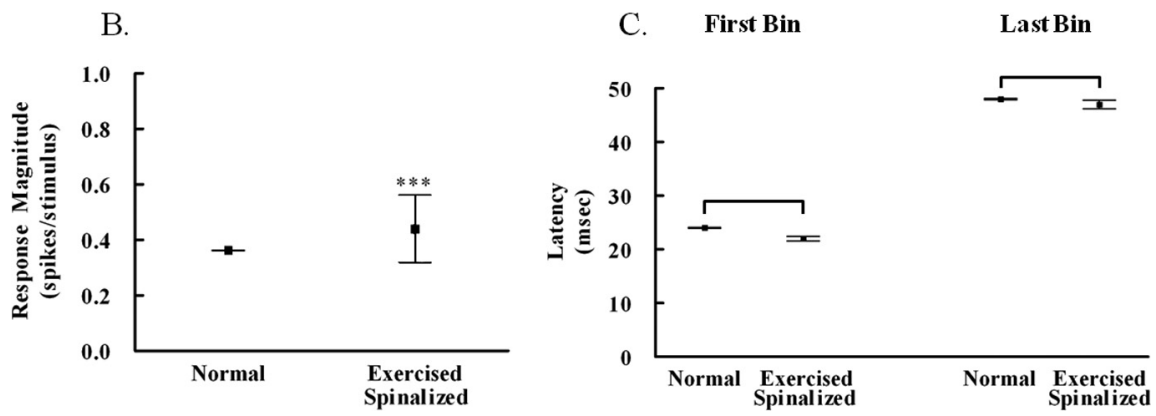

Figure 3. Exercise increased the response magnitude of cells in the infragranular hindlimb cortex to forelimb stimulation in spinalized rats. $A$, Examples of peristimulus time histograms showing the responses of four infragranular hindlimb cells recorded simultaneously from each of three stimulus locations: forepaw digit 2 (FD2), forepaw palm pad 5 (FPL5), and forelimb (FL). $\boldsymbol{B}$, The average response magnitude of infragranular cells within the hindlimb somatosensory cortex of exercised spinalized rats $(0.4408 \pm 0.12$ spikes/stimulus, $N=10$ rats $)$ was greater than that of normal rats $(0.3625 \pm 0.002$ spikes/stimulus, $N=9$ rats $)$; ${ }^{* * *} p<0.001$. C, No differences were observed between the first bin latency of exercised spinalized rats ( $22 \pm 0.4 \mathrm{~ms}$ ) versus that of normals $(24 \pm 0.08 \mathrm{~ms})$ or the last bin latency of exercised spinalized rats $(47 \pm 0.8 \mathrm{~ms})$ versus that of normals ( $48 \pm 0.13 \mathrm{~ms})$. Error bars indicate SEM.

$(n=3)$ were selected for serotonergic staining (5-HT). Segments of the cord rostral (as control) and caudal to the transection segment was sectioned transversely and stained with a polyclonal antibody to 5-HT. Frozen sections mounted on slides were incubated at $4^{\circ} \mathrm{C}$ with the primary antibody (diluted 1:40,000; Immunostar) for $16 \mathrm{~h}$, with biotinylated goat anti-rabbit IgG for $2 \mathrm{~h}$ and with avidin-biotinylated horseradish peroxidase complex for $2 \mathrm{~h}$, as specified by the manufacturer (ABC Standard Kit; Vector Laboratories). Peroxidase reactivity was visualized with $0.05 \%$ diaminobenzidine tetrahydrochloride and $0.01 \%$ hydrogen peroxide in $0.05 \mathrm{~mm}$ Tris buffer. No staining for $5-\mathrm{HT}$ was found caudal to the transection.

\section{Results}

Treadmill exercise induces novel organization in the hindlimb somatosensory cortex after neonatal midthoracic transection

As expected, some cells in hindlimb cortex of normal adult rats respond to stimulation of the forelimbs, presumably via intracortical connections (Chapin et al., 1987). A midthoracic transection eliminates hindlimb input to the somatosensory cortex while leaving forelimb input intact and thus would be expected to eliminate the responsiveness of cells in the somatosensory cortex to stimulation of peripheral areas below the level of the transection. To assess the effect of exercise on the responsiveness of cells in the hindlimb somatosensory cortex to cutaneous forelimb stimulation, acute single-neuron mappings were performed in three groups of animals: normal control $(N=4)$, neonatally spinalized
Last Bin

rats that did not receive treadmill exercise $(N=5)$, and neonatally spinalized rats that received treadmill exercise $(N=5)$. For each penetration, we averaged the percentage of responding cells across groups. There was a significant group difference between the average percentage of cells that responded to cutaneous stimulation (ANOVA, $p<0.02$ ). In fact, the average percentage of cells in the hindlimb cortex responding to stimulation in the exercised spinalized rats $(38.7 \pm 16 \%$ of 294 cells $)$ was significantly greater than either that of the normal $(8.3 \pm 4.5 \%$ of 311 cells $)$ or spinalized $(7.7 \pm 8.4 \%$ of 150 cells $)$ rats (Tukey post hoc, $p<0.05$ for both).

There were also notable differences on a cortical layer-by-layer basis between the animal groups (Fig. 2). Since cells were not recorded from every cortical layer during each electrode penetration, the numbers of penetrations for which cells were recorded from for each animal are reported separately for the supragranular, granular, and infragranular layers (Fig. $2 A-C$ ). As expected, in normal rats, a small percentage of cells in all layers of the hindlimb somatosensory cortex responded to stimulation of the forelimb, [supragranular $(3 \pm 4 \%)$, granular $(11 \pm 10 \%)$, infragranular $(11 \pm 5 \%)]$. The spinalized rats showed similar percentages of cells that responded to sensory stimulation in all cortical layers [supragranular (0\%), granular $(17 \pm 7 \%)$, infragranular $(6 \pm 3 \%)]$ (Fig. $2 D-F)$. Since the cells recorded from the spinalized rats also did not respond to cutaneous stimulation of the hindlimbs, these cells would have been silenced in response to the neonatal spinalization. This is in agreement with several studies on the effects of spinalization on the responsiveness of cells in the affected region of the cortex.

A different picture emerged when we examined the cells recorded from the exercised spinalized animals. Treadmill exercise markedly increased the percentages of cells that responded to cutaneous forelimb stimulation in the supragranular and infragranular hindlimb somatosensory cortices of spinalized rats [supragranular $(46 \pm 5 \%)$, infragranular $(50 \pm 5 \%)$ ] when compared with those of normal [supragranular (3 $\pm 4 \%)$, infragranular $(11 \pm 5 \%)]$ or neonatally spinalized rats that did not receive exercise [supragranular $(0 \%)$, infragranular $(6 \pm$ $3 \%)$ ] (Fig. 2D,F). There was no noticeable difference between the percentage of responding cells in the granular layer of the exercised spinalized rats $(21 \pm 6 \%)$ when compared with the percentages of responding cells in the granular layers of the normal $(11 \pm 10 \%)$ or spinalized rats that did not receive exercise $(17 \pm 7 \%)$ (Fig. $2 E)$. In fact, the exercised spinalized rats had a significantly greater proportion of cells in the hindlimb cortex responding to sensory stimulation of the forelimbs in the supragranular and infragranular layers (two proportions test, $p<$ 0.001 for both) but not in the granular layer $(p=0.07)$. Therefore, treadmill exercise induced a novel organization in the extra- 
granular layers of the hindlimb somatosensory cortex after neonatal T8/T9 transection.

Treadmill exercise increases the magnitude of the response of hindlimb infragranular neurons to sensory stimuli

To determine if exercise also altered the magnitude of the response of extragranular cells to sensory stimuli in the spinalized exercised group, a second study was then performed using two additional groups of animals: spinalized rats that received exercise $(N=10)$ and normal rats $(N=9)$. Infragranular cells in the hindlimb somatosensory cortex were studied using multiple, single-neuron-recording techniques from arrays of microwire electrodes chronically implanted into the infragranular hindlimb somatosensory cortex (see Materials and Methods). The response of each cell to cutaneous stimulation of each peripheral location was quantified from the cell's peristimulus time histogram (Fig. $3 A$ ). In general, the infragranular hindlimb cells in the exercisedspinalized rats responded with greater magnitude than those of the normal rats.

As expected, some cells in the infragranular hindlimb somatosensory cortex of normal rats responded to cutaneous stimulation of the forelimb. The average magnitude of these responses (response magnitude, $0.3625 \pm 0.002$ spikes/stimulus) was less than that of cells recorded from the forelimb somatosensory cortex to the same stimulation (response magnitude, $0.6266 \pm 0.001$ spikes/stimulus; $p<0.001$ ), and the latency was longer (first bin latency, $24 \pm 0.08 \mathrm{~ms}$ for hindlimb somatosensory cortex cells compared with $18 \pm 0.47 \mathrm{~ms}$ for forelimb somatosensory cortex cells; $p<0.001$ ) consistent with our previous study (Moxon et al., 2008). It has been suggested that the responsiveness of cells recorded from the hindlimb cortex to cutaneous forelimb stimulation is mediated by intracortical connections from the forelimb somatosensory cortex to the hindlimb somatosensory cortex (Chapin et al., 1987), and this could account for the delay.

Cells of the hindlimb somatosensory cortex of exercisedspinalized rats showed an increase in the average magnitude of response (response magnitude, $0.4408 \pm 0.012$ spikes/stimulus vs response magnitude, $0.3625 \pm 0.002$ spikes/stimulus; $p<$ 0.001) (Fig. 3B) but without changes in the latency measures (first bin latency, $22 \pm 0.4 \mathrm{~ms}$ and last bin latency, $47 \pm 0.8 \mathrm{~ms}$ for exercised spinalized rats; first bin latency, $24 \pm 0.08 \mathrm{~ms}$ and last bin latency, $48 \pm 0.13 \mathrm{~ms}$ for normal rats) (Fig. $3 C$ ). Therefore, the exercise-induced increase in the percentage of cells that respond to sensory stimulation after neonatal SCI is accompanied by an increase in the magnitude of the response of these cells.

\section{Weight-supported stepping is correlated with the response magnitude of cells in the infragranular hindlimb somatosensory cortex}

We next evaluated whether the increased response magnitude of cells in the hindlimb somatosensory cortex was correlated with the ability of exercised spinalized rats to perform WSS on the treadmill (Fig. 4). Normally, a small number of neonatally spinalized rats develop some ability to support their own weight while locomoting on a treadmill (Miya et al., 1997; Giszter et al., 1998; Kao et al., 2006). The average response magnitude of the hindlimb cells was positively correlated to the \%WSS $(r=0.814$, $p<0.01$ ), suggesting that the extent to which the hindlimb somatosensory cortex can respond to cutaneous forelimb sensory stimulation may be related to the ability of the rat to take more weight-supported steps.
A.
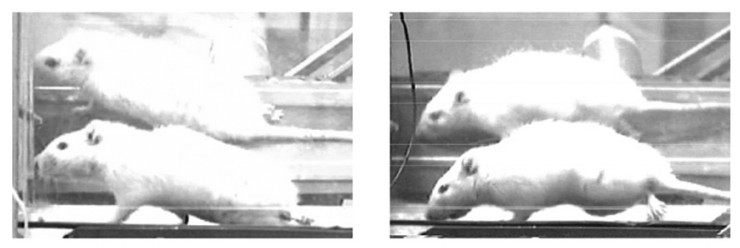

B.

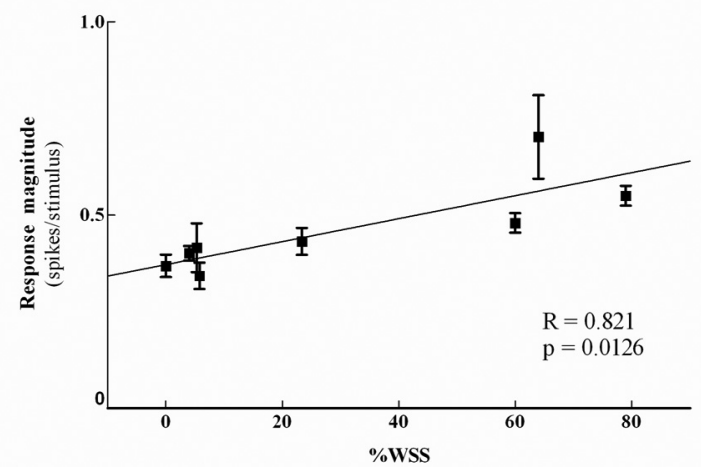

Figure 4. The response magnitude of cells in the infragranular layer of the hindlimb cortex correlated with percentage weight-supported steps in exercised spinalized rats. $A$, Photographs of two adult rats with neonatal T8/T9 transections performing treadmill exercise (a mirror is placed behind the treadmill to ensure all activity of the hindlimbs is captured). On the left is one exercised spinalized rat exhibiting no weight support. The hindquarters are dragged on the surface of the treadmill. On the right is an exercised spinalized rat exhibiting a weightsupported step. The hindlimbs are supporting the hindquarters above the surface of the treadmill. $\boldsymbol{B}$, The average weight-supported steps for eight rats were obtained after electrode implantation in the hindlimb somatosensory cortex. Each box represents different days of the sensory maps. Five rats had undergone two different days of sensory maps, whereas three rats had one sensory map. The average response magnitude of the cells were positively correlated with \%WSS $\left({ }^{* *} p<0.01\right)$.

Treadmill exercise restores percentage of responding cells in the forelimb somatosensory cortex

To assess the effect of exercise after neonatal spinalization on the percentage of responding cells in the forelimb somatotopic representation (an area of cortex not directly affected by the injury), acute, single-neuron mappings of the forelimb somatosensory cortex were performed on normal $(N=4)$, neonatally spinalized rats that that did not receive exercise $(N=4)$ and neonatally spinalized rats that received exercise $(N=5)$. Surprisingly, there was a significant group difference for the average percentage of cells that responded to cutaneous stimulation (ANOVA, $p<$ $0.01)$. For the spinalized rats, there was a significant decrease in the percentage of cells responding to sensory stimulation (35.3 \pm $12.6 \%$ of 240 cells) compared with those of the normal $(72.3 \pm$ $13 \%$ of 140 cells) or exercised spinalized $(76.5 \pm 9.7 \%$ of 138 cells) rats (Tukey post hoc, $p<0.02$ ). We suspect that spinalized animals have reduced locomotion within their cages, resulting in the reduced sensory responsiveness compared with normal animals and that the treadmill exercise replaced the reduced locomotion of the spinalized animals, therefore restoring the sensory responsiveness of the cells. However, further study is required to confirm this.

These differences in the forelimb somatosensory cortex could also be assessed on a layer-by-layer basis (Fig. 5). Since cells were not recorded from every layer during each penetration, the numbers of penetrations for which cells were recorded for each animal are reported separately for the supragranular, granular, and infragranular layers (Fig. 5A-C). Across all the three cortical layers, the normal rats and exercised spinalized rats had similar percentages of cells that responded to sensory stimulation [normal: su- 

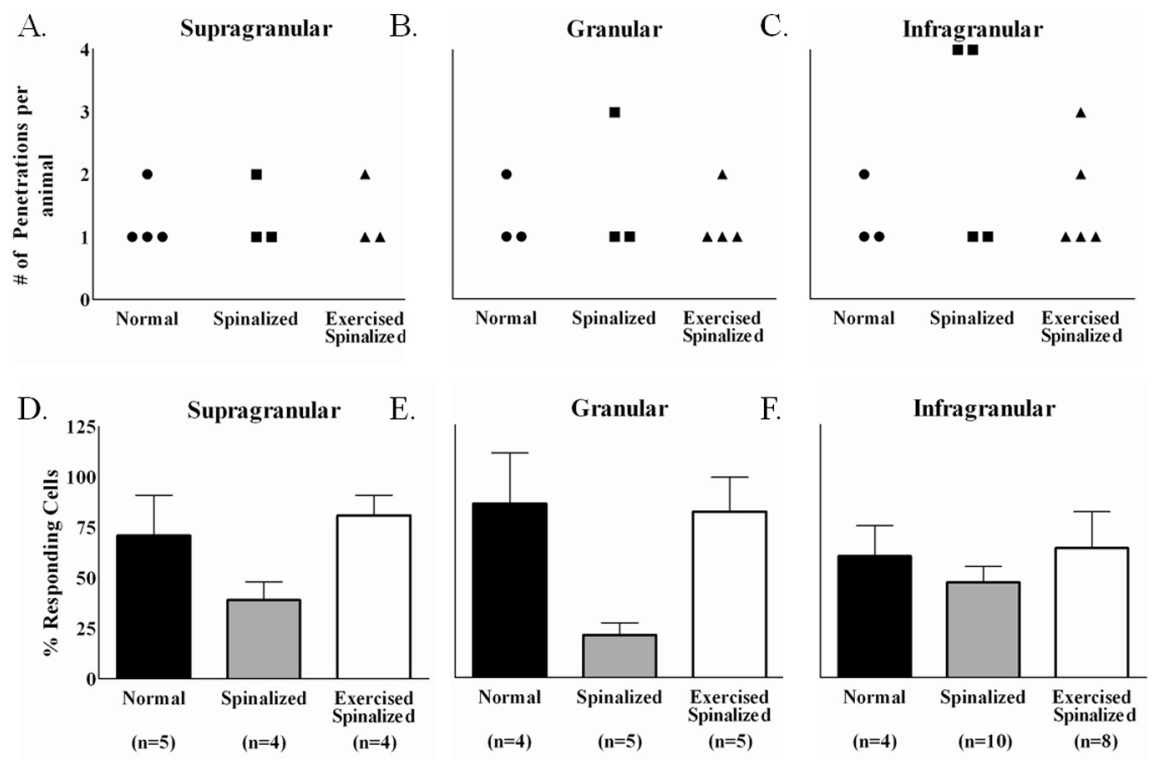

Figure 5. Exercise restored the percentage of cells that responded to sensory stimulations in the forelimb somatosensory cortex of spinalized rats. Data were collected from four normal, four spinalized, and five exercised spinalized rats. $\boldsymbol{A}-\boldsymbol{C}$, The numbers of electrode penetrations within the forelimb somatosensory cortex from which cells were detected are shown for each animal (circles represent normal animals, squares represent spinalized animals, and triangles represent exercised spinalized animals). $\boldsymbol{D}-\boldsymbol{F}$, The averages of the percentages of cells that responded to sensory stimuli for each penetration ( $n=$ total number of penetrations) were plotted as a per layer basis for each animal group. As expected, a high percentage of cells in all cortical layers of the forelimb cortex responded to sensory stimulation of the forelimb [supragranular ( $71 \%$ of 42 cells), granular ( $86 \%$ of 21 cells), and infragranular (60\% of 77 cells)]. Neonatal spinalization decreased the percentages of sensory responsive cells in all layers of the forelimb cortex [supragranular ( $39 \%$ of 31 cells), granular ( $21 \%$ of 28 cells), and infragranular ( $47 \%$ of 181 cells)] when compared with those of the normal rats. Exercise restored the percentages of sensory responsive cells in all cortical layers of spinalized rats, comparable with those of normals [supragranular ( $81 \%$ of 21 cells), granular ( $82 \%$ of 27 cells), and infragranular (64\% of 90 cells)]. Error bars indicate SEM.

pragranular $(71 \pm 20 \%)$, granular $(86 \pm 25 \%)$, infragranular $(60 \pm 15 \%)$; exercised spinalized: supragranular $(81 \pm 10 \%)$, granular $(82 \pm 17 \%)$, infragranular $(64 \pm 18 \%)$ ] (Fig. 5D-F). However, the percentages of sensory responsive cells recorded from neonatally spinalized rats were markedly reduced across all three cortical layers [supragranular $(39 \pm 9 \%)$, granular $(21 \pm$ $6 \%)$, infragranular $(46 \pm 8 \%)]$. This reduction was significant across all the layers (two proportions test, $p<0.01$ for supragranular, $p<0.001$ for granular, and $p<0.05$ for infragranular). Therefore, neonatal transection at the T8/T9 level diminishes the somatotopic representation in the forelimb somatosensory cortex, and this can be reversed by treadmill exercise. These results suggest that for adult rats with neonatal thoracic transections, our paradigm of 3 min per day, $5 \mathrm{~d}$ per week of treadmill exercise for 6-8 months is sufficient to compensate, at the supraspinal level, for their lack of mobility.

\section{Treadmill exercise increases the response magnitude of cells in the infragranular forelimb somatosensory cortex after neonatal spinalization}

Despite the fact that there were no differences in the percentage of responding cells between normal and exercised spinalized rats, treadmill exercise significantly increased the response magnitude of forelimb somatosensory cortex cells in exercised spinalized rats $(N=5)$ to sensory stimulation of the forelimb periphery compared with that of normal animals $(N=4)(p<0.001)$. The response of each cell to stimulation of each location was quantified from the cell's peristimulus time histogram (Fig. 6A).

The average response magnitude of infragranular cells in the forelimb somatosensory cortex of exercised spinalized rats (re- sponse magnitude, $1.49 \pm 0.07$ spikes/ stimulus) was greater than that of the infragranular cells in normal rats (response magnitude, $0.6266 \pm 0.001$ spikes/stimulus) (Fig. 6B). The change in response magnitude did not affect the first bin latency (for exercised spinalized rats, $18 \pm$ $0.47 \mathrm{~ms}$; for normal rats, $17 \pm 0.02 \mathrm{~ms}$ ) (Fig. 6C) but was accompanied by a change in the last bin latency (for exercised spinalized rats, $48 \pm 0.94 \mathrm{~ms}$; for normal rats, $39 \pm 0.05 \mathrm{~ms})(p<0.001)$. This created an overall increase in the duration of the response of forelimb cells to forelimb stimulation in exercised-spinalized rats compared with that of normal rats. The increased response magnitude of these forelimb somatosensory cells was not correlated to the increase in \%WSS. However, the response magnitude of these forelimb cells was significantly correlated to the increased response magnitude of hindlimb cells (data not shown). Therefore, interactions between the hindlimb somatosensory cortex and the forelimb somatosensory cortex could be responsible for the increased duration of the response of forelimb cells that is evident by an increase in response magnitude of forelimb cells of exercised spinal rats compared with normal rats.

\section{Discussion}

Our studies suggest that cortical plasticity after spinal cord injury can be induced by sensorimotor stimulation, such as that derived from treadmill exercise. In the hindlimb somatosensory cortex of spinalized rats that did not receive treadmill exercise, very few cells responded to cutaneous stimulation of the forelimb, similar to what is seen in normal animals. After daily treadmill exercise, however, cells in the hindlimb somatosensory cortex of the spinalized rats were more likely to respond to cutaneous forelimb sensory stimulation. These cells also responded with more spikes per stimulus than cells recorded from the hindlimb somatosensory cortex of normal animals. Therefore, cortical plasticity is not prevented by the spinal cord injury itself but requires sensorimotor stimulation to induce changes in the somatotopic map of the cortex.

Importantly, the increase in response magnitude of hindlimb infragranular neurons after exercise was correlated to the \%WSS. Since the percentage of cells responding to sensory stimulation was not correlated (data not shown), these results suggest that exercise increases the number of cells responding to the sensory stimulation regardless of its impact on weight-supported stepping. This increase in number of cells responding to a stimulus, combined with a correlation between number of spikes per stimulus and \%WSS, suggest that for a given stimulus, there is a substantial increase in neuronal activity for animals that achieve the highest levels of weight-supported stepping.

The changes in the hindlimb cortex after neonatal thoracic transection and treadmill exercise occurred only in the extragranular layers. Since the granular layer of the hindlimb somatosensory cortex was not affected by exercise, consistent with previous multiunit mapping studies (Jain et al., 2003), 
thalamocortical processing is unlikely to be markedly affected by the exercise. The fact that there was a significant effect of exercise on the extragranular layers of the hindlimb somatosensory cortex suggests that changes in corticocortical activity are responsible. The functional organization of the cortex supports the idea that sensory information is processed between layers within a column before it is transmitted to other columns via the extragranular layers (Silva and Koretsky, 2002). However, a recent study in primate using a unilateral cervical spinal cord injury demonstrated reorganization in the thalamus that paralleled that of the cortex after $\sim 2$ years (Jain et al., 2008). Therefore, a thalamocortical influence on the effects seen in the cortex cannot be ruled out without further study.

Surprisingly, the spinalized rats that did not receive treadmill exercise had fewer cells in the forelimb somatosensory cortex that responded to stimulation of the forelimb compared with normal rats. This could be attributable to some developmental change after the spinalization; however, it is most likely because of the greater restriction of their movements when compared with the normal and exercised spinalized rats. Thus, sensory input to the intact forelimb is reduced. Previous studies have demonstrated a similar effect in that this type of reduced sensory input has been shown to reduce the area of the forelimb cortex that responds to sensory stimulation (Langlet et al., 1999).

As might be expected, this reduction effect was reversed after the spinalized rats participated in treadmill exercise. There-

fore, the amount of exercise these spinalized rats were exposed to in this study was likely to be sufficient to normalize their activity to levels comparable with those of normal rats. Furthermore, the magnitude of the response of infragranular forelimb cells to forelimb stimulation was greater in exercise-spinalized animals than normal animals. This increase in response magnitude was accompanied by an increase in duration of the response. Although neither of these measures correlated to behavioral outcome in these rats, the increase in response magnitude of the infragranular forelimb cells was correlated to the increase in the response magnitude of the infragranular hindlimb cells suggesting significant interactions between these two areas of the brain. Recent evidence has suggested that molecular mechanisms within the deprived and adjacent cortical representations of SCI rats may decrease inhibitory neurite outgrowth, thus enhancing intracortical connections between these two representations (Endo et al., 2007).

Despite the fact that our results support corticocortical changes, we cannot determine if these changes were attributable to changes in synaptic efficacy, sprouting, or the formation of new connections across cortical regions. In normal animals after exercise, in vitro studies of local circuits to identify mechanisms for changes in cortical organization suggested that the ability of cells to alter synaptic efficacy depends on levels of incoming ac- tivity by increasing synaptic efficacy attributable to a general excitation of the cortical cells (Abraham and Bear, 1996; Hickmott and Merzenich, 2002). Peripheral injury studies to identify the mechanism for cortical plasticity of affected regions of the cortex also support corticocortical plasticity (Donoghue and Sanes, 1988; Wang et al., 1995; Lane et al., 1999). Finally, synaptic plasticity has been shown in the motor cortex of adult rats with $\mathrm{C} 4$ overhemisections. In these cells, dendritic spine length increased and spine density decreased within $7 \mathrm{~d}$ but returned to normal by day 28 after hemisection "indicating a more immature and modifiable pattern of synaptic connectivity” (Kim et al., 2006). However, the spinal cord-injured rats of that study did not receive any explicit exercise protocol. It remains unclear if more long-term changes in the cortex would have been observed if the rats had received any form of exercise.

When neonatally spinalized rats are trained to walk on a treadmill, they can develop high levels of weight-supported stepping (Miya et al., 1997). It had been presumed that this occurs because of reorganization within the spinal cord of neonatally injured rats that is unavailable to adult injured rats. However, follow-up studies (Giszter et al., 1998) demonstrated that treadmill training induced novel organization in the motor cortex of rats spinalized as neonates, namely, the increased cortical representation of a subset of the axial musculature. This novel organization pattern 
was related to functional recovery. Although the precise mechanism by which axial muscles are recruited in this animal model is unknown, Giszter et al. (1998) postulated that although the hindquarters continued to operate mainly autonomously from the forelimbs, the increased control of the axial muscles could improve balance, stabilization, steering control, and some propulsive coupling to the caudal axial and hindlimb system. Recent work (Giszter et al., 2007) analyzing the ability of these animals to maintain stance in the presence of controlled perturbations demonstrated that the strategy of these animals relied completely on their forelimb. Essentially, the forelimbs worked to isolate the hindlimbs from the perturbations and reduced the load on the hindlimbs. These two factors may combine to allow local pattern generators below the level of the lesion to provide weightsupported stepping in response to local reflexes without instabilities and stance failure, which is now completely controlled by the forelimbs. Our data now extend these results to demonstrate that weight-supported stepping may also take advantage of increased representation of the forelimbs in the sensory cortex. Moreover, our result that the number of weight-supported steps was significantly correlated to the magnitude of the neuronal responses in the novel cortical representation of the forelimbs suggests that this enhanced sensory processing may also contribute to the isolation of the hindlimbs, contributing to the improved balance and making it easier for these animals to take weight-supported steps. Therefore, in rats with complete spinal cord transection, exercise alters both the sensory and motor organization of the cortex, and this novel organization may, in part, contribute to the increased weight-supported steeping of animals with spinal cord injury.

\section{References}

Abraham WC, Bear MF (1996) Metaplasticity: the plasticity of synaptic plasticity. Trends Neurosci 19:126-130.

Cai LL, Fong AJ, Otoshi CK, Liang Y, Burdick JW, Roy RR, Edgerton VR (2006) Implications of assist-as-needed robotic step training after a complete spinal cord injury on intrinsic strategies of motor learning. J Neurosci 26:10564-10568

Chapin JK (1986) Laminar differences in sizes, shapes, and response profiles of cutaneous receptive fields in the rat SI cortex. Exp Brain Res 62:549-559.

Chapin JK, Sadeq M, Guise JL (1987) Corticocortical connections within the primary somatosensory cortex of the rat. J Comp Neurol 263:326-346.

Chau CW, McKinley PA (1991) Chronological observations of primary somatosensory cortical maps in kittens following low thoracic (T12) spinal cord transection at 2 weeks of age. Somatosens Mot Res 8:355-376.

Donoghue JP, Sanes JN (1988) Organization of adult motor cortex representation patterns following neonatal forelimb nerve injury in rats. J Neurosci 8:3221-3232.

Endo T, Spenger C, Tominaga T, Brené S, Olson L (2007) Cortical sensory map rearrangement after spinal cord injury: fMRI responses linked to Nogo signaling. Brain 130:2951-2961.

Florence SL, Boydston LA, Hackett TA, Lachoff HT, Strata F, Niblock MM (2001) Sensory enrichment after peripheral nerve injury restores cortical, not thalamic, receptive field organization. Eur J Neurosci 13:1755-1766.

Foffani G, Tutunculer B, Moxon KA (2004) Role of spike timing in the forelimb somatosensory cortex of the rat. J Neurosci 24:7266-7271.

Friedberg MH, Lee SM, Ebner FF (1999) Modulation of receptive field properties of thalamic somatosensory neurons by the depth of anesthesia. J Neurophysiol 81:2243-2252.

Friel KM, Heddings AA, Nudo RJ (2000) Effects of postlesion experience on behavioral recovery and neurophysiologic reorganization after cortical injury in primates. Neurorehabil Neural Repair 14:187-198.
Giszter SF, Kargo WJ, Davies M, Shibayama M (1998) Fetal transplants rescue axial muscle representations in M1 cortex of neonatally transected rats that develop weight support. J Neurophysiol 80:3021-3030.

Giszter SF, Davies MR, Graziani V (2007) Motor strategies used by rats spinalized at birth to maintain stance in response to imposed perturbations. J Neurophysiol 97:2663-2775.

Hickmott PW, Merzenich MM (2002) Local circuit properties underlying cortical reorganization. J Neurophysiol 88:1288-1301.

Jain N, Florence SL, Kaas JH (1995) Limits on plasticity in somatosensory cortex of adult rats: hindlimb cortex is not reactivated after dorsal column section. J Neurophysiol 73:1537-1546.

Jain N, Diener PS, Coq JO, Kaas JH (2003) Patterned activity via spinal dorsal quadrant inputs is necessary for the formation of organized somatosensory maps. J Neurosci 23:10321-10330.

Jain N, Qi HX, Collins CE, Kaas JH (2008) Large-scale reorganization in the somatosensory cortexand thalamus after sensory loss in the macaque monkey. J Neurosci 28:11042-11060.

Kao T, Shumsky JS, Jacob-Vadakot S, Himes BT, Murray M, Moxon KA (2006) Role of the 5-HT2C receptor in improving weight-supported stepping in adult rats spinalized as neonates. Brain Res 11:159-168.

Kim BG, Dai HN, McAtee M, Vicini S, Bregman BS (2006) Remodeling of synaptic structures in the motor cortex following spinal cord injury. Exp Neurol 198:401-415.

Lane RD, Stojic RS, Killackey HP, Rhoades RW (1999) Source of inappropriate receptive fields in cortical somatotopic maps from rats that sustained neonatal forelimb removal. J Neurophysiol 81:625-633.

Langlet C, Canu MH, Falempin M (1999) Short-term reorganization of the rat somatosensory cortex following hypodynamia-hypokinesia. Neurosci Lett 266:145-148.

Leiser SC, Moxon KA (2006) Relationship between physiological response type (RA and SA) and vibrissal receptive field of neurons within the rat trigeminal ganglion. J Neurophysiol 95:3129-3145.

Leiser SC, Moxon KA (2007) Responses of trigeminal ganglion neurons during natural whisking behaviors in the awake rat. Neuron 53:117-133.

McKinley PA, Swyter E (1989) Progressive changes in somatosensory cortical maps in 6-week-old kittens cord-transected at T12. Brain Res 484:378-382.

Miya D, Giszter S, Mori F, Adipudi V, Tessler A, Murray M (1997) Fetal transplants alter the development of function after spinal cord transection in newborn rats. J Neurosci 17:4856-4872.

Moxon KA, Hale LL, Aguilar J, Foffani G (2008) Responses of infragranular neurons in the rat primary somatosensory cortex to forepaw and hindpaw tactile stimuli. Neuroscience 156:1083-1092.

Paxinos G, Watson C (2007) The rat brain in stereotaxic coordinates, Ed 6. Boston: Academic/Elsevier.

Ramanathan D, Conner JM, Tuszynski MH (2006) A form of motor cortical plasticity that correlates with recovery of function after brain injury. Proc Natl Acad Sci U S A 103:11370-11375

Shumsky JS, Kao T, Amato N, Simansky K, Murray M, Moxon KA (2005) Partial 5-HT(1A) receptor agonist activity by the 5-HT(2C) receptor antagonist SB 206,553 is revealed in rats spinalized as neonates. Exp Neurol 191:361-365.

Silva AC, Koretsky AP (2002) Laminar specificity of functional MRI onset times during somatosensory stimulation in rat. Proc Natl Acad Sci U S A 99:15182-15187.

Tutunculer B, Foffani G, Himes BT, Moxon KA (2006) Structure of the excitatory receptive fields of infragranular forelimb neurons in the rat primary somatosensory cortex responding to touch. Cereb Cortex 16:791-810.

Wang X, Merzenich MM, Sameshima K, Jenkins WM (1995) Remodelling of hand representation in adult cortex determined by timing of tactile stimulation. Nature 378:71-75.

Weidner N, Ner A, Salimi N, Tuszynski MH (2001) Spontaneous corticospinal axonal plasticity and functional recovery after adult central nervous system injury. Proc Natl Acad Sci U S A 98:3513-3518.

Wong-Riley M (1979) Changes in the visual system of monocularly sutured or enucleated cats demonstrable with cytochrome oxidase histochemistry. Brain Res 171:11-28. 